\title{
Proceso cognitivo y metacognitivo en prescolares con riesgo de dificultades de aprendizaje de las matemáticas según sus padres
}

\author{
María Llobet Piñas \\ al202464@uji.es \\ Jose Antonio Rodríguez Niño \\ jose.rodriguez@uji.es \\ Lorena Zorrilla Silvestre \\ al106987@uji.es
}


I. Resumen

Deficiencias en algunos componentes de funcionamiento ejecutivo, como control emocional, memoria de trabajo o iniciativa han sido relacionados con baja evolución en el aprendizaje de las matemáticas. El funcionamiento ejecutivo puede ser evaluado mediante pruebas neuropsicológicas en situaciones de laboratorio o también mediante cuestionarios. El Behavior Rating Inventory of Executive Function (BRIEF, Gioia, G.; Isquith, P.; Guy S. y Kenworthy L., 2000) es uno de los instrumentos más idóneos para evaluar el funcionamiento ejecutivo en niños en edades prescolares al permitir un análisis en ambientes naturales a partir de la información aportada por los padres o los profesores. El propósito de este trabajo es examinar si existen diferencias entre las puntuaciones en funcionamiento ejecutivo de niños con y sin riesgo de déficit en el aprendizaje de las matemáticas (DAM), según sus padres. El estudio se realizó con 65 alumnas y alumnos de la provincia de Castellón, con un rango de edad entre 5 y 6 años. Los resultados muestran diferencias significativas entre ambos grupos en funcionamiento ejecutivo en los índices generales del cuestionario y en algunas de las habilidades específicas analizadas. Estos resultados plantean la posibilidad de manifestaciones diferenciales de las funciones ejecutivas en diferentes contextos.

\section{Introducción}

Las competencias matemáticas básicas, los factores motivacionales y el funcionamiento ejecutivo, son los tres factores más influyentes que han sido reflejados en los diversos estudios que se han realizado sobre el aprendizaje de las matemáticas.

Diferentes planteamientos centrados específicamente en habilidades o competencias matemáticas básicas coexisten en la actualidad para la explicación de las dificultades de aprendizaje de las matemáticas. Desde el modelo piagetiano la habilidad matemática depende del descubrimiento por parte del niño de una serie de operaciones lógicas básicas: seriación, clasificación, conservación e inclusión. Este planteamiento ha recibido el soporte de estudios que han mostrado la relación de estas operaciones lógicas con el logro matemático que alcanza el niño.

Para muchos investigadores las operaciones lógicas no son condiciones previas, necesarias y suficientes para el aprendizaje de las matemáticas. Desde una perspectiva neopiagetina creen que lo específico para el 
aprendizaje de la aritmética son las habilidades de conteo que incluye tanto aspectos cognitivos (conceptual counting knowledge) como procedimentales (procedural counting knowledge).

Un enfoque más reciente y alternativo para explicar los problemas en el cálculo es el planteado por Butterworth (2003). Este autor, tras una revisión de trabajos sobre desarrollo infantil, neuropsicológicos, genéticos y de neuroimagen, concluye que la idea de numerosidad, entendida como la habilidad para estimar intuitivamente el número de elementos de un conjunto y sus relaciones (subitizing), es la que mejor explica el desarrollo de las habilidades aritméticas y sus dificultades.

Por otro lado parece claro que las creencias atribucionales, o causas percibidas de éxito y fracaso, son una variable que tiene repercusiones directas en el rendimiento escolar en general y específicamente en el área de las matemáticas. Asimismo, las actitudes que tengan los alumnos hacia la materia, es decir, si se ven o no a sí mismos capaces de aprender y resolver tareas matemáticas y si las consideran útiles y con sentido, pueden jugar un papel importante en el rendimiento en matemáticas.

Por último y en el que nos centramos en este trabajo, las funciones ejecutivas.

El ámbito de las funciones ejecutivas suele definirse, en términos globales, sobre un amplio abanico de operaciones cognitivas que guardan entre sí una relación. En general, dentro de este concepto se incluyen habilidades vinculadas a la capacidad de organizar y planificar una tarea, seleccionar apropiadamente los objetivos, iniciar un plan y sostenerlo en la mente mientras se ejecuta, inhibir las distracciones, cambiar de estrategias de modo flexible si el caso lo requiere, autorregular y controlar el curso de la acción para asegurarse que la meta propuesta esté en vías de lograrse, etc. En síntesis, inhibición, cambio, control emocional, iniciativa, memoria de trabajo, organización de materiales, monitorización y planificación y organización constituyen requisitos importantes para resolver problemas de manera eficaz y eficiente.

Las funciones ejecutivas parecen ser indispensables para el logro de metas escolares y laborales ya que coordinan y organizan procesos cognoscitivos básicos, como son la memoria y la percepción requeridas para un comportamiento propositivo. Es por eso que la alteración de estas funciones puede limitar la capacidad del individuo para mantener una vida independiente y productiva, aún si otras habilidades cognoscitivas se encuentren intactas. 
Las investigaciones de Bull y otros (2008); Brock y otros (2009) y la más reciente y más relevante, de Clark y otros (2010), han encontrado relaciones significativas entre medidas de planificación, control inhibitorio, flexibilidad cognitiva o memoria de trabajo en último curso de prescolar y el posterior rendimiento en matemáticas.

También cabe destacar que Toll y otros (2010) utilizando un diseño longitudinal han encontrado que, entre un conjunto de funciones ejecutivas, las tres tareas de memoria de trabajo predecían la pertenencia al grupo de estudiantes con DAM, mientras que solamente una tarea de inhibición y ninguna de flexibilidad cognitiva demostraron el mismo poder predictivo. Informando de un poder explicativo superior de las funciones ejecutivas, especialmente de la memoria de trabajo, sobre los aprendizajes matemáticos.

\section{Objetivos}

El principal objetivo de la investigación es realizar un análisis exhaustivo de las diferentes variables de funcionamiento ejecutivo que predicen mejor las dificultades matemáticas en niños en edad prescolar en el contexto familiar valorado por los padres. Pues el funcionamiento ejecutivo tiene peso en el aprendizaje de las matemáticas desde el inicio del desarrollo del niño y sobre todo en el futuro rendimiento, sin embargo no todas están de acuerdo en qué variables afectan con mayor intensidad en este hecho.

\section{Material y método}

Para comprobar estas diferencias se ha utilizado el cuestionario conductual de funciones ejecutivas (BRIEF; Behavior Rating Inventory of Executive Function) de Giogia, Isquith, Guy y Kenworthy (2000). EI BRIEF fue diseñado, desarrollado y baremado por autores americanos, y presenta dos formas principales de aplicación: un cuestionario aplicado a padres, y otro aplicado a profesores. Ambos son cuestionarios breves que se cumplimentan en 10-15 minutos y que consisten en indicar, el nivel de problematicidad que presenta el sujeto evaluado en las diferentes áreas del funcionamiento ejecutivo. Este instrumento es una de las pruebas de referencia en este campo y proporciona información sobre los principales dominios (índice de regulación comportamental e índice de metacognición), así como una puntuación global de función ejecutiva.

Esta prueba mide aspectos del funcionamiento ejecutivo que no están 
recogidos en otras pruebas más tradicionales, y su aparición obedece a la necesidad de encontrar métodos de carácter ecológico válidos de los aspectos más molares, conductuales y cotidianos de las funciones ejecutivas en los niños. Los resultados obtenidos en los cuestionarios muestran un perfil de afectación en las distintas funciones ejecutivas, y resulta útil para la evaluación de trastornos como el déficit de atención/hiperactividad (TDAH) o los trastornos de conducta de origen orgánico. Miranda (2001) y Miranda y otros (2005 y 2009).

La muestra la componen 65 niños de preescolar $5,3^{\text {er }}$ curso del segundo ciclo de educación infantil, de 5 años y 10 meses de media de edad, 29 de los cuales son niñas y 36 niños. Los colegios son de la provincia de Castellón en concreto 4 de Castellón capital, 3 de la Vall d'Uxó y 1 de Moncofar, de los cuales 7 son públicos y 1 concertado.

Se seleccionaron aquellos niños que su rendimiento matemático no fuera explicado por ninguna otra patología, ya fuese retraso mental, trastorno del desarrollo... se les aplicó 2 subpruebas del WIPPSI, que nos permite obtener un $\mathrm{Cl}$ equivalente y descartar niños que estén por debajo de la media. Para la clasificación de los niños en los grupos de con o sin riesgo en matemáticas se ha utilizado un cuestionario para la/el maestra/o, además de una prueba de matemáticas que permite comparar las competencias matemáticas ya adquiridas, el TEDI-MATH.

El procedimiento seguido para la recogida y selección de la muestra fue en primer lugar dar información y realizar la petición formal al colegio, en concreto equipo directivo y orientador, en segundo lugar nos entrevistamos con las maestras de Infantil 5 años que no tuvieron ningún problema en ofrecerse voluntarias e informaron a los padres, ya que estos deben autorizar de manera formal la participación de su hijo en la muestra. Posteriormente seleccionamos la muestra, recogimos la información necesaria de las maestras y se entregó el cuestionario para los padres. Nos entrevistamos con los padres que necesitaron ayuda para rellenar el cuestionario y finalmente corregimos los test y obtuvimos los resultados, que posteriormente discutimos.

Para el análisis de los datos utilizamos un programa informático, SPSS, realizamos en primer lugar la prueba de normalidad de KolmogorovSmirnov, y posteriormente dada la normalidad de la distribución de todas las variables se procedió con la comparación de la media mediante la prueba Tstudent. 
EI BRIEF nos proporciona un índice global de funcionamiento ejecutivo donde se puede apreciar la diferencia entre las medias, siendo superior la puntuación para niños con riesgo.

\section{Resultados}

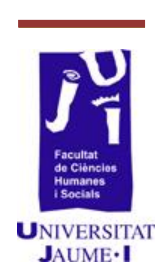

Tabla 1. Estadísticos de grupo y prueba T

\begin{tabular}{|c|c|c|c|c|c|}
\hline \multicolumn{2}{|c|}{ GRUPO } & \multirow{2}{*}{$\begin{array}{c}\text { Media } \\
16,63 \\
16,29 \\
\end{array}$} & \multirow{2}{*}{$\begin{array}{c}\begin{array}{c}\text { Desviación } \\
\text { típ. }\end{array} \\
4,605 \\
3,486 \\
\end{array}$} & \multirow{2}{*}{$\frac{\mathbf{t}}{\mathrm{t}}$} & \multirow{2}{*}{$\begin{array}{c}\begin{array}{c}\text { Sig. } \\
\text { (bilateral) }\end{array} \\
, 731\end{array}$} \\
\hline $\begin{array}{l}\text { BRIEF: PADRES } \\
\text { INHIBICIÓN } \\
\end{array}$ & $\begin{array}{l}\text { NIÑOS CON RIESGO } \\
\text { NIÑOS SIN RIESGO }\end{array}$ & & & & \\
\hline $\begin{array}{l}\text { BRIEF: PADRES } \\
\text { CAMBIO }\end{array}$ & $\begin{array}{l}\text { NIÑOS CON RIESGO } \\
\text { NIÑOS SIN RIESGO }\end{array}$ & $\begin{array}{l}12,63 \\
11,83 \\
\end{array}$ & $\begin{array}{l}2,456 \\
2,561 \\
\end{array}$ & 1,291 & 201 \\
\hline $\begin{array}{l}\text { BRIEF: PADRES } \\
\text { CONTROL } \\
\text { EMOCIONAL }\end{array}$ & $\begin{array}{l}\text { NIÑOS CON RIESGO } \\
\text { NIÑOS SIN RIESGO }\end{array}$ & $\begin{array}{l}16,37 \\
17,34\end{array}$ & $\begin{array}{l}3,709 \\
3,955\end{array}$ & $-1,026$ & 309 \\
\hline $\begin{array}{l}\text { BRIEF: PADRES } \\
\text { INICIATIVA }\end{array}$ & $\begin{array}{l}\text { NIÑOS CON RIESGO } \\
\text { NIÑOS SIN RIESGO }\end{array}$ & $\begin{array}{l}12,63 \\
11,23 \\
\end{array}$ & $\begin{array}{l}2,282 \\
2,486 \\
\end{array}$ & 2,374 & ,021 \\
\hline $\begin{array}{l}\text { BRIEF: PADRES } \\
\text { MEMORIA TRABAJO }\end{array}$ & $\begin{array}{l}\text { NIÑOS CON RIESGO } \\
\text { NIÑOS SIN RIESGO }\end{array}$ & $\begin{array}{l}17,13 \\
12,94 \\
\end{array}$ & $\begin{array}{l}3,730 \\
2,980 \\
\end{array}$ & 4,947 & ,000 \\
\hline $\begin{array}{l}\text { BRIEF: PADRES } \\
\text { PLANIFICACIÓN } \\
\text { ORGANIZACIÓN }\end{array}$ & $\begin{array}{l}\text { NIÑOS CON RIESGO } \\
\text { NIÑOS SIN RIESGO }\end{array}$ & $\begin{array}{l}19,83 \\
16,40\end{array}$ & $\begin{array}{l}3,788 \\
3,070\end{array}$ & 3,971 & ,000 \\
\hline $\begin{array}{l}\text { BRIEF: PADRES } \\
\text { ORGANIZACIÓN } \\
\text { MATERIALES }\end{array}$ & $\begin{array}{l}\text { NIÑOS CON RIESGO } \\
\text { NIÑOS SIN RIESGO }\end{array}$ & $\begin{array}{l}10,27 \\
9,91\end{array}$ & $\begin{array}{l}3,723 \\
3,166\end{array}$ & ,407 & ,685 \\
\hline $\begin{array}{l}\text { BRIEF: PADRES } \\
\text { MONITORIZACIÓN }\end{array}$ & $\begin{array}{l}\text { NIÑOS CON RIESGO } \\
\text { NIÑOS SIN RIESGO }\end{array}$ & $\begin{array}{l}14,63 \\
12,89 \\
\end{array}$ & $\begin{array}{l}2,671 \\
2,654 \\
\end{array}$ & 2,637 & ,011 \\
\hline $\begin{array}{l}\text { BRIEF: PADRES } \\
\text { ÍNDICE GLOBAL } \\
\text { COMPUESTO (CEG) }\end{array}$ & $\begin{array}{l}\text { NIÑOS CON RIESGO } \\
\text { NIÑOS SIN RIESGO }\end{array}$ & $\begin{array}{l}120,13 \\
108,83\end{array}$ & $\begin{array}{l}20,099 \\
17,022\end{array}$ & 2,424 & ,019 \\
\hline
\end{tabular}

En términos generales se puede observar también que para todas las variable las medias son mayores para niños con riesgo, excepto para control emocional que es mayor en el caso de los niños sin riesgo. Las diferencias observadas no son grandes pero si lo son en iniciativa, planificación, monitorización y especialmente en memoria de trabajo. 
Las diferencias son significativas. En concreto son significativas en iniciativa, memoria de trabajo, planificación y monitorización. Además para el índice global también se observa que las diferencias son significativas.

Concretando un poco todo lo visto anteriormente, cabe decir que los resultados observados apuntan a los resultados de investigaciones como la de Toll, Van der Ven, Kroesbergen y Van Luit (2010) que informan de que la memoria de trabajo predecirá mejor que otras variables las dificultades en el aprendizaje de las matemáticas. Y no se confirma los resultados de otras investigaciones que señalan que la mayor parte de las variables que intervienen en el funcionamiento ejecutivo explican o predicen las futuras dificultades en las matemáticas por igual.

\section{Discusión y conclusiones}

El índice global que nos proporciona el BRIEF nos dice que sí que existen diferencias entre las medias de los niños con y sin riesgo, siendo mayor en el caso segundo.

Para las demás variables también se observa estas diferencias en mayor o menor grado. Cabe decir que destacan estas diferencias en iniciativa, memoria de trabajo, planificación y organización y monitorización. Por otro lado se puede comprobar que para todos los casos estas diferencias son significativas exceptuando el control emocional. Es importante destacar también que estos resultados podrían no deberse a la casualidad en tanto y cuanto dispusiéramos de una muestra un tanto mayor.

Por lo que desde un punto de vista mas cualitativo y por tanto analítico de los resultados se puede concretar que el funcionamiento ejecutivo sí que apunta a la predicción de las posibles dificultades en las matemáticas como se informaba en estudios anteriores como el de Clark, Pritchard y Woodward (2010). En este estudio se afirmaba que las medidas de planificación, control inhibitorio, flexibilidad cognitiva o memoria, si mostraban relación con las posibles discalculias, por lo que podemos ampliar diciendo que también las medidas tomadas para iniciativa, organización y planificación.

Concretamos tras una larga discusión que esto podía ser debido a al menos 3 situaciones diferentes. La primera y más evidente es que los padres y los maestros valoran de manera diferente, siendo en los primero el criterio más laxo y en los segundos un criterio más técnico o estricto. La segunda razón va en la línea de las tareas que se valoran, que valorándose 
las mismas variables la tareas o acciones son completamente diferentes y derivada de esta razón tenemos la tercera y última, las exigencias establecidas para las tareas en el aula y por el maestros pueden llegar a ser de un nivel más alto y con menos ayudas que en las tareas realizadas en el contexto familiar.

Por eso queremos dar importancia al hecho de que a la hora de realizar una evaluación se deben tener en cuenta los diferentes ámbitos o contextos en los que se desarrolla el niño.

Finalmente decir que los estudios realizados que tienen en cuenta los tres factores que intervienen en el aprendizaje de las matemáticas, competencias, motivación y funcionamiento ejecutivo, son pocos. Además de haber observado a lo largo de la revisión y de la realización de este trabajo la importancia del funcionamiento ejecutivo en el aprendizaje de las matemáticas.

Por ello concluimos que la aplicación de un programa que se incluya en el currículum para el entrenamiento de las habilidades o funciones ejecutivas debe ser algo que esté presente en los docentes. Programas como el de Adele Diamond, Tools of the Mind: selfregulation thought a curriculum, es un programa para la primera infancia basado en la investigación sobre la emergencia y la estimulación de las funciones autorregulatorias, a través de un currículo educativo de base vigotskiana. A través de una serie de rigurosas pruebas experimentales, Tools of the Mind -que recibió un premio de UNESCO a la excelencia como propuesta curricular-, ha demostrado tener un impacto significativo en el desarrollo de la autorregulación. El estudio también encontró que estas ganancias en la autorregulación, se asocian a mayores puntuaciones en el desempeño escolar durante las fases de alfabetización temprana y de adquisición de conocimientos matemáticos.

\section{Bibliografía}

AMES, C., (1992), "Classrooms: Goals, structures, and student motivation», Journal of Educational Psychology, Vol 84(3), Sep 1992, 261-271. doi: 10.1037/00220663.84.3.261

ANDERSSON, U. (2008). "Working memory as a predictor of written Arithmetical skills in children: The importance of central executive Functions». British Journal of Educational Psychology, 78, 181-203.

BROCK L., L. y otros (2009), "The contributions of 'hot' and 'cool' executive function to children's academic achievement, learning-related behaviors, 
and engagement in kindergarten", Early Childhood Research Quarterly, 24, Issue 3, 3rd Quarter, 337-349, ISSN 0885-2006, 10.1016/j.ecresq.2009.06.001.

BULL, R. y otros (2008). "Short-term memory, working memory, and executive functioning in preschoolers: Longitudinal predictors of mathematical achievement at age 7 years». Developmental Neuropsychology, 33, 205-228.

BUTTERWORTH, B (2003): Dyscalculia Screener, NFER-Nelson, London.

ClaRK, C. A. C. y otros (2010). "Preschool executive functioning abilities predict early mathematics achievement». Developmental Psychology, 46(5), 1176-1191. doi: http://dx.doi.org/10.1037/a0019672

ESPY, K. A. y otros (2004), "The contribution of executive functions to emergent mathematic skills in preschool children». Developmental Neuropsychology, 26, 465-486.

MiRANDA CASAS, A. (2001): Trastornos por déficit de atención con hiperactividad: una guía práctica. Málaga: Aljibe.

Miranda CASAS, A. y otros (2005), "Nuevas tendencias en la evaluación de las dificultades de aprendizaje de las matemáticas. El papel de la metacognición». Revista de Neurología, 40, 97-102.

MiRANDA CASAS, A. y otros (2009), «Habilidades matemáticas y funcionamiento ejecutivo de niños con trastorno por déficit». Psicothema. Vol. 21, no 1, 63-69

PASSOLUNGHI, M. C. y otros (2007). "The precursors of mathematics learning: Working memory, phonological ability and numerical competence». Cognitive Development, 22, 165-184.

PiAget J. y R. García (1987): Hacia una lógica de significaciones. Barcelona: Gedisa.

Nicholls, J. G. (1984): Psychological Review.

SASTRE-RIBA, S. (2006), "Condiciones tempranas del desarrollo y el aprendizaje: el papel de las funciones ejecutivas». Revista de neurología, 42 (Supl 2), S143-S151

St Clair-Thompson, H. L., y S. E. Gathercole (2006). «Executive functions and achievements in school: Shifting, updating, inhibition, and working memory». Quarterly Journal of Experimental Psychology, 59, 745-759.

TOLL, SYLKE W. M. y otros (2011). "Executive Functions as Predictors of Math Learning Disabilities». Hammill Institute on Disabilities. J Learn Disabil, 44, 521. 
RODRÍGUEZ NIÑO, J. A., (EN PRENSA), «Funcionamiento ejecutivo valorado por los maestros en niños de educación infantil con diferentes niveles de matemáticas», Universidad Jaume I, Castellón.

VAN DER SLUIS, S. y otros (2004), "Inhibition and shifting in children with learning deficits in arithmetic and reading». Journal of Experimental Child Psychology, 87, 239-266.

VUKOVIC, R. K. Y L. S. SIEGEL (2010), «Academic and cognitive characteristics of persistent mathematics difficulty form first through fourth grade». Learning Disabilities Research \& Practice, 25, 25-38. 